\title{
A Compact Antenna for Ultrawide-Band Applications
}

\author{
Nader Behdad, Student Member, IEEE, and Kamal Sarabandi, Fellow, IEEE
}

\begin{abstract}
A novel compact and ultrawide-band (UWB) antenna is presented in this paper. The basis for achieving such an UWB operation is through proper magnetic coupling of two adjacent sectorial loop antennas in a symmetrical arrangement. A large number of coupled sectorial loop antennas (CSLA) with different geometrical parameters are fabricated and their measured responses are used to experimentally optimize the geometrical parameters of the antenna for achieving the maximum bandwidth. Through this optimization process an antenna with a VSWR of lower than $2.2\left(S_{11}<-8.5 \mathrm{~dB}\right)$ across an 8.5:1 frequency range is designed. The maximum dimension of this antenna is smaller than $0.37 \lambda_{0}$ at the lowest frequency of operation and provides an excellent polarization purity. Furthermore, the antenna exhibits a relatively consistent radiation pattern. Modified versions of the CSLA are also designed to reduce the overall metallic surface and weight of the antenna while maintaining its wide-band characteristics. This allows modifying its dimensions to design low frequency light-weight UWB antennas.
\end{abstract}

Index Terms-Broadband antennas, loop antennas, ultrawideband (UWB) antennas.

\section{INTRODUCTION}

A few decades after the early investigations on ultrawide-band (UWB) wireless systems, they have found a wide range of applications including ground penetrating radars, high data rate short range wireless local area networks, communication systems for military, and UWB short pulse radars for automotive and robotics applications to name a few [1]. Such systems require antennas that are able to operate across a very large bandwidth with consistent polarization and radiation patterns parameters over the entire band. A number of techniques have been developed in past to design antennas with wide-band impedance matched characteristics. Traveling wave antennas and antennas with topologies that are invariant by rotation are inherently wide-band and have been extensively used [2]-[4]. Self complementary concept [5] is another method that can provide a constant input impedance irrespective of frequency provided that the size of the ground plane for the slot segment of the antenna is large and an appropriate self-complementary feed can also be designed. Theoretically, the input impedance of self-complementary antennas is $186 \Omega$ and cannot be directly matched to standard transmission lines. Another draw back of self-complementary structures is that they can not be printed on a dielectric substrate, since the dielectric constant of the substrate perturbs the self-complementary condition. Another

Manuscript received October 1, 2004; revised January 19, 2005. This work was supported by EMAG Technologies, Incorporated, Ann Arbor, MI.

The authors are with The Radiation Laboratory, Department of Electrical Engineering and Computer Science, The University of Michigan, Ann Arbor, MI 48109-2122 USA (e-mail: behdad@engin.umich.edu).

Digital Object Identifier 10.1109/TAP.2005.850750 technique for designing wide-band antennas is to use multiresonant radiating structures. Log-periodic antennas, microstrip patches with parasitic elements, and slotted microstrip antennas for broad-band and dual-band applications are examples of this category [2], [6]-[9].

The electric dipole and monopole above a ground plane are perhaps the most basic types of antennas available. Variations of these antennas have recently been introduced for obtaining considerably larger bandwidths than the traditional designs [10]-[12]. Impedance bandwidth characteristics of circular and elliptical monopole plate antennas are examined in [10]. Wide-band characteristics of rectangular and square monopole antennas are studied in [11] and a dielectric loaded wide-band monopole is investigated in [12]. A draw back of these types of antennas is that the antenna polarization as a function of frequency changes. In this paper, a new type of single-element wide-band antenna is proposed that can provide wider bandwidth and consistent polarization over the frequency range that the antenna is impedance matched. The antenna is composed of two parallel coupled sectorial loop antennas (CSLA) that are connected along an axis of symmetry. The geometrical parameters of this antenna are experimentally optimized and it is shown that the antenna can easily provide a wide-band impedance match over an 8.5:1 frequency range. The gain and radiation patterns across the frequency range of operation remain almost constant particularly over the first two octaves of its return loss bandwidth. The antenna geometry is then modified in order to reduce its size and weight without compromising its bandwidth. This helps fabrication and installation of the antenna operating for lower frequency applications such as ground penetrating radars or broadcast television where the physical size of the antenna is large.

In what follows, first the principle of operation of the CSLA is introduced, then the design and experimental optimization of the geometrical parameters of the CSLA is presented. Finally the geometry of this optimized CSLA is modified to reduce the height, weight, and overall metallic surface of the antenna and two modified CSLAs are introduced and the radiation parameters of the optimized antennas across their entire bands of operation are presented and discussed.

\section{COUPLED Sectorial LoOP ANTENNA}

\section{A. Principle of Operation}

The topology of a sectorial loop antenna (SLA) composed of an arch and two sectors is shown in Fig. 1(a). The input impedance of this relatively narrow band antenna, $Z_{s}$, is a function of three geometrical parameters, $R_{\text {in }}, R_{\text {out }}$, and $\alpha$. Such loop antennas, similar to a circular loop, present a strong antiresonance (also called parallel-resonance) when the antenna circumference is about $C \approx 0.5 \lambda$. In other words, the equivalent 


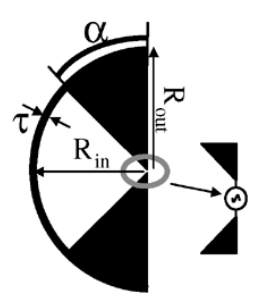

(a)

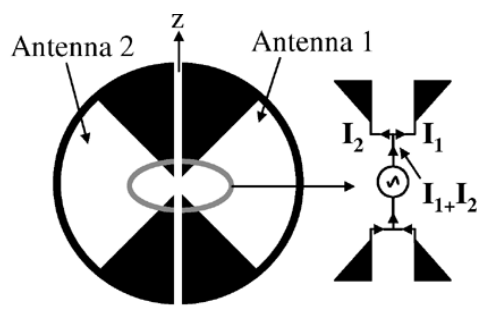

(b)

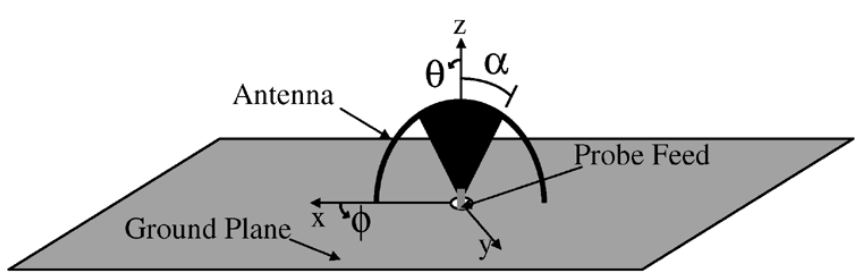

(c)
Fig. 1. (a) Topology of a sectorial loop antenna. (b) Topology of a coupled sectorial loop antenna (CSLA). (c) Half of a CSLA above a ground plane is used and the antenna is fed with a coaxial probe to eliminate the need for using a BALUN.

circuit of the antenna is a high-Q parallel $R L C$ and, hence, $Z_{s}$ is inductive immediately below and capacitive immediately above this antiresonance. For $C>0.5 \lambda$, other antiresonances are not very sharp (equivalent circuits are low $\mathrm{Q}$ parallel $R L C$ ) and the variations of the input reactance are not very large. If these variations are minimized, the antenna bandwidth can be increased significantly. One way of controlling the input impedance is by connecting two identical antennas in parallel and controlling the mutual coupling between them. Here, this is accomplished by connecting two identical SLAs in parallel as shown in Fig. 1(b). In this case, because of the symmetry, the input currents $I_{1}$ and $I_{2}$ are equal but the direction of magnetic field of loop \# 1 (Antenna \#1) is opposite to that of loop \#2 (Antenna \#2) and therefore, the magnetic flux of the two loops link strongly, giving rise to a strong mutual coupling. The geometrical parameters can be varied to control the mutual coupling as a function of frequency. For the two-port system of antennas shown in Fig. 1(b), the following equations can be written:

$$
\left\{\begin{array}{l}
V_{1}=Z_{11} I_{1}+Z_{12} I_{2} \\
V_{2}=Z_{21} I_{1}+Z_{22} I_{2}
\end{array}\right.
$$

where, $V_{1}, I_{1}, V_{2}$, and $I_{2}$ are the voltages and currents at the input ports of the Antenna \#1 and Antenna \#2, respectively, [see Fig. 1(b)]. $Z_{11}\left(Z_{22}\right)$ is the input impedance of the Antenna \#1 (Antenna \#2) in the presence of Antenna \#2 (Antenna $\# 1$ ) and when it is open circuited. $Z_{21}$ and $Z_{12}$ represent the mutual coupling between the two antennas. In this case, reciprocity mandates that $Z_{12}=Z_{21}$ and the symmetry requires that $Z_{11}=Z_{22}$. When the two antennas are connected in parallel and fed with a single source, as is shown in Fig. 1(b), we will have $V_{1}=V_{2}$ and as a consequence of symmetry $I_{1}=I_{2}=I$. The input impedance of the antenna can then be obtained from

$$
Z_{\text {in }}=\frac{1}{2}\left(Z_{11}+Z_{12}\right)
$$

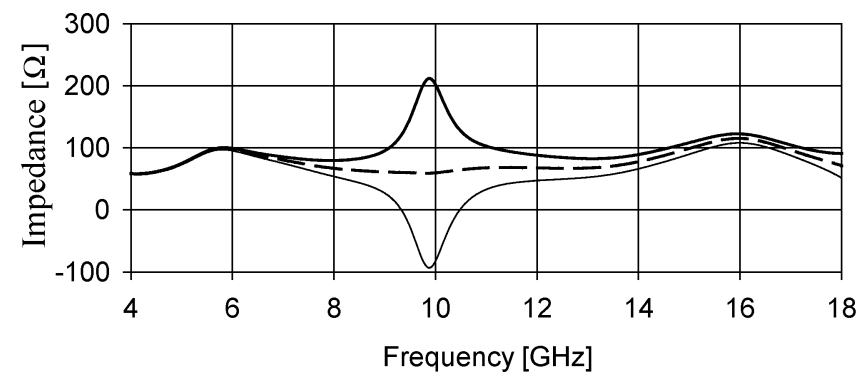

(a)

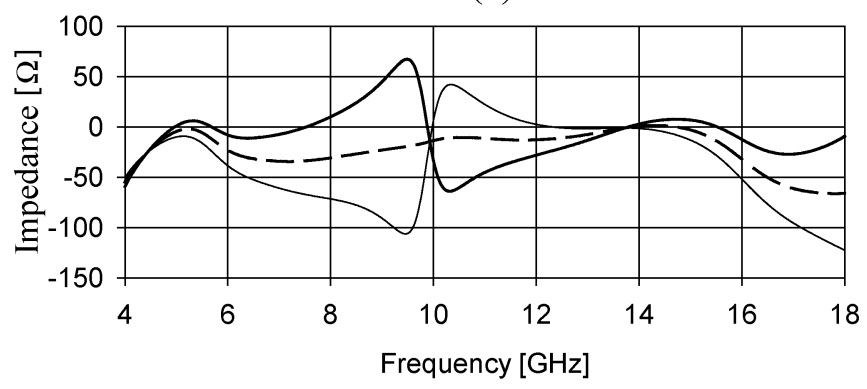

(b)

Fig. 2. Self and mutual impedances of two SLAs that are $\mathrm{d}=0.01 \lambda_{0}$ apart and have $R_{\text {in }}=13 \mathrm{~mm}, R_{\text {out }}=14 \mathrm{~mm}$, and $\alpha=60^{\circ}$. (a) Real and (b) imaginary part. Thick solid line: self impedance, thin solid line: mutual impedance, and dashed line: input impedance as defined by (1).

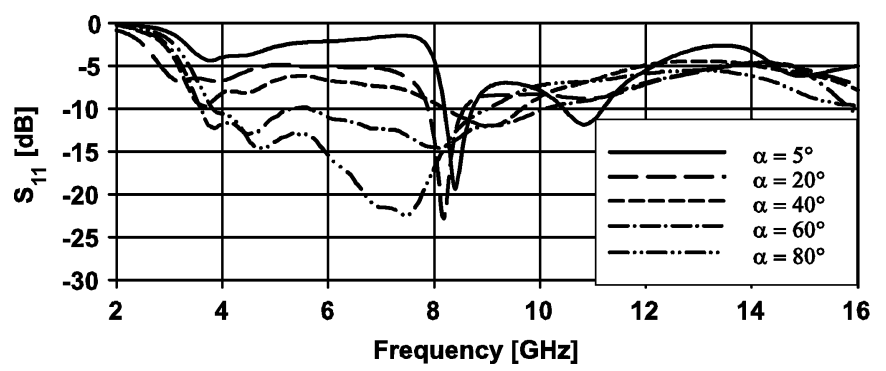

(a)

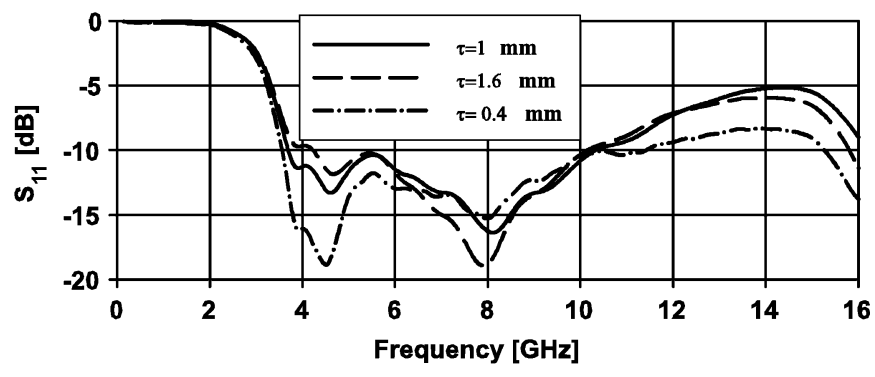

(b)

Fig. 3. Measured $S_{11}$ values of a number of CSLAs used in the experimental optimization process. (a) $R_{\text {in }}=13 \mathrm{~mm}, R_{\text {out }}=14 \mathrm{~mm}$, and different $\alpha$ values. (b) $R_{\mathrm{av}}=13.5 \mathrm{~mm}, \alpha=60^{\circ}$, and different $\tau$ values $\left(\tau=R_{\text {out }}-\right.$ $\left.R_{\text {in }}\right)$.

In order to achieve a wide-band operation spectral variations of $Z_{11}$ and $Z_{12}$ must counteract each other. That is, when the real (imaginary) part of $Z_{11}$ increases with frequency the real (imaginary) part of $Z_{12}$ should decrease so that their average remains constant. This can be accomplished by optimizing the geometrical parameters of the antenna. Here, $Z_{11}$ and $Z_{12}$ are obtained by calculating the input and mutual impedances of two adjacent SLA [as shown in Fig. 1(b)] using full-wave FDTD 

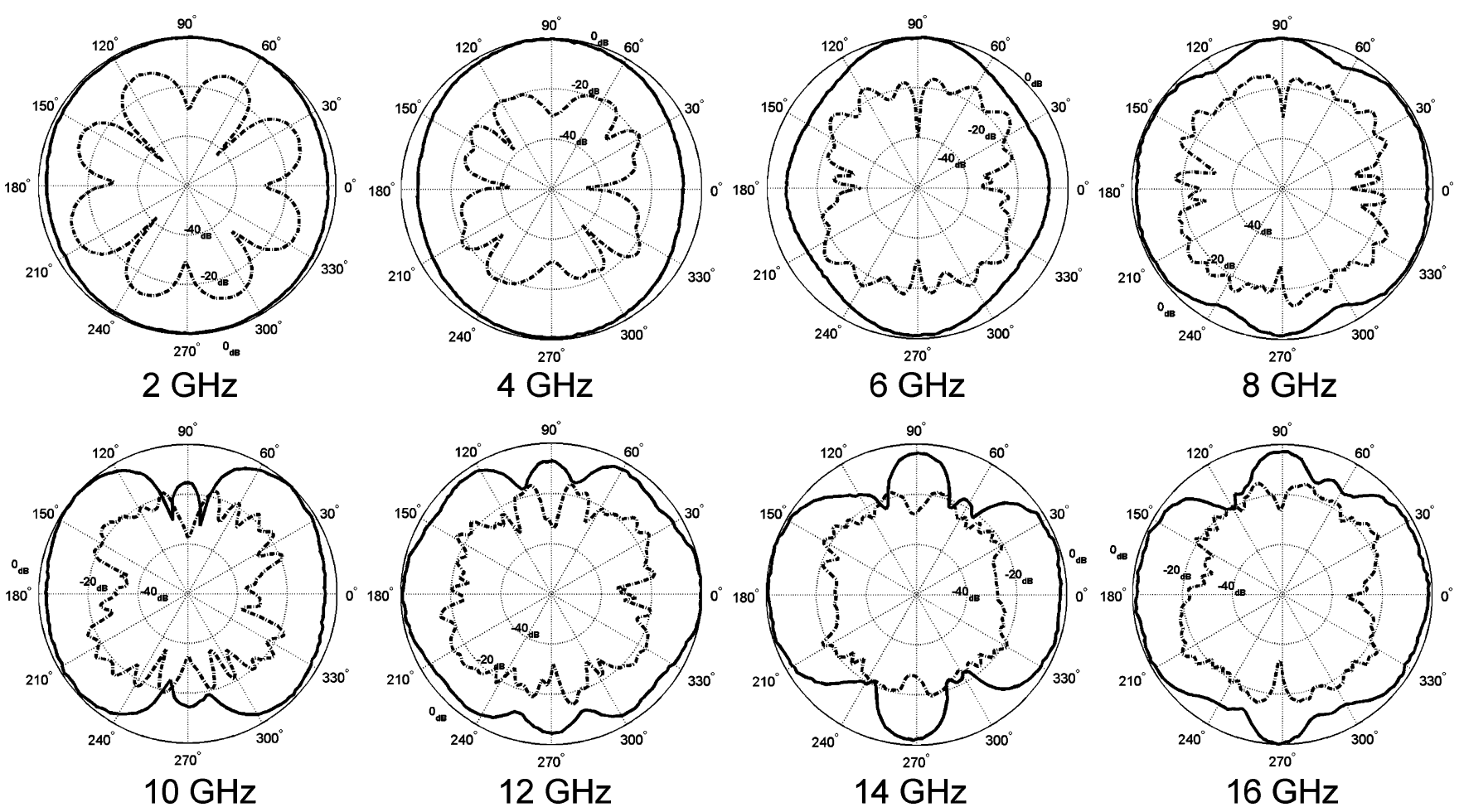

Fig. 4. Measured radiation patterns of the CSLA in Section II in the azimuth plane. The solid line is co-pol $\left(E_{\theta}\right)$ and the dash-dotted line is the cross-pol $\left(E_{\phi}\right)$ component.

simulations. The simulations are performed and the self and mutual impedances of two adjacent SLAs with $R_{\text {in }}=13 \mathrm{~mm}$ and $R_{\text {out }}=14 \mathrm{~mm}$ and different values of $\alpha$ are calculated. In these simulations, the antennas are placed at a distance of $d=0.01 \lambda_{\max }$ apart ( $\lambda_{\max }$ is the wavelength at lowest frequency of operation). From the simulations' results, it is observed that as $\alpha$ increases from $0^{\circ}$ to $90^{\circ}$, the variations in the real and imaginary parts of $Z_{11}$ and $Z_{12}$ start to counteract each other and a relatively constant input impedance is achieved. This phenomenon is more clearly observed for $\alpha$ values in the range of $40^{\circ} \leq \alpha \leq 80^{\circ}$. Fig. 2 shows the real and imaginary parts of $Z_{11}$ and $Z_{12}$ for two adjacent SLAs when $R_{\text {in }}=13 \mathrm{~mm}$, $R_{\text {out }}=14 \mathrm{~mm}$, and $\alpha=60^{\circ}$. As can be observed from this figure, the variations cancel each other over a wide bandwidth giving rise to an almost constant input impedance. In the next sub-section, an experimental approach is followed to find the optimum $\alpha$ value that provides the largest bandwidth.

\section{B. Sensitivity Analysis}

An experimental sensitivity analysis is carried out to determine the optimum geometrical parameters of the proposed CSLA. The antenna is composed of two sectorial loops connected in parallel along the axis of symmetry ( $z$-axis) as shown in Fig. 1(b). The three parameters that affect the antenna response are the inner and outer radii of the loop, $R_{\text {in }}$ and $R_{\text {out }}$, and the angle $\alpha$ as shown in Fig. 1. The lowest frequency of operation is determined by the overall, average effective circumference of each sectorial loop as expressed by the following approximate formula:

$$
f_{l}=\frac{2 c}{(\pi-\alpha+2) \sqrt{\epsilon_{\text {eff }}}\left(R_{\text {in }}+R_{\text {out }}\right)}
$$

where $\epsilon_{\text {eff }}$ is the effective dielectric constant of the antenna's surrounding medium, c is the speed of light, and $R_{\mathrm{in}}, R_{\text {out }}$, and $\alpha$ are the geometrical parameters of the antenna. In this formula the average circumference of the SLA is considered to be $(\pi-\alpha) R_{\mathrm{av}}+2 R_{\mathrm{av}}$, where the factor 2 comes from the fact that each SLA is composed of an arc and two straight arms each having a length of $R_{\mathrm{av}}$. The effective dielectric constant can easily be calculated using quasi-static method of moments or finite difference analysis. Choosing the lowest frequency of operation, the average radius of the loop, $R_{\mathrm{av}}=\left(R_{\mathrm{in}}+R_{\text {out }}\right) / 2$, can be determined from (2). Therefore the parameters that remain to be optimized are $\alpha$ and $\tau=\left(R_{\text {out }}-R_{\text {in }}\right)$. In order to obtain the optimum value of $\alpha$, nine different antennas with $\alpha$ values ranging from $5^{\circ}$ up to $80^{\circ}$ with $R_{\text {in }}=13 \mathrm{~mm}$ and $R_{\text {out }}=14 \mathrm{~mm}$ were fabricated and their $S_{11}$ as a function of frequency were measured. Since the antenna topology shown in Fig. 1(b) needs a balanced feed, half of the antenna along the plane of zero potential $(z=0)$ over a ground plane fed by a coaxial cable is used [as shown in Fig. 1(c)]. In order to circumvent the fabrication process, the antennas are fabricated using printed circuit technology and printed on a $3 \mathrm{~cm} \times 1.5 \mathrm{~cm}$ thin dielectric substrate with dielectric constant of $\epsilon_{r}=3.4$ and thickness of $500 \mu \mathrm{m}$ and are mounted on a $10 \mathrm{~cm} \times 10 \mathrm{~cm}$ ground plane. For brevity, only measured $S_{11}$ values for $\alpha=5^{\circ}$, $20^{\circ}, 40^{\circ}, 60^{\circ}, 80^{\circ}$ are presented in Fig. 3(a). It is shown that as $\alpha$ increases from $5^{\circ}$ to $90^{\circ}$, the impedance bandwidth increases and reaches its maximum at $\alpha=60^{\circ}$ at which an impedance bandwidth ranging from $3.7-10 \mathrm{GHz}$ is achieved. The next step in the experimental optimization of the geometrical parameters of CSLA is to find the optimum value of the arch thickness, $\tau$. This is done by fabricating antennas with 

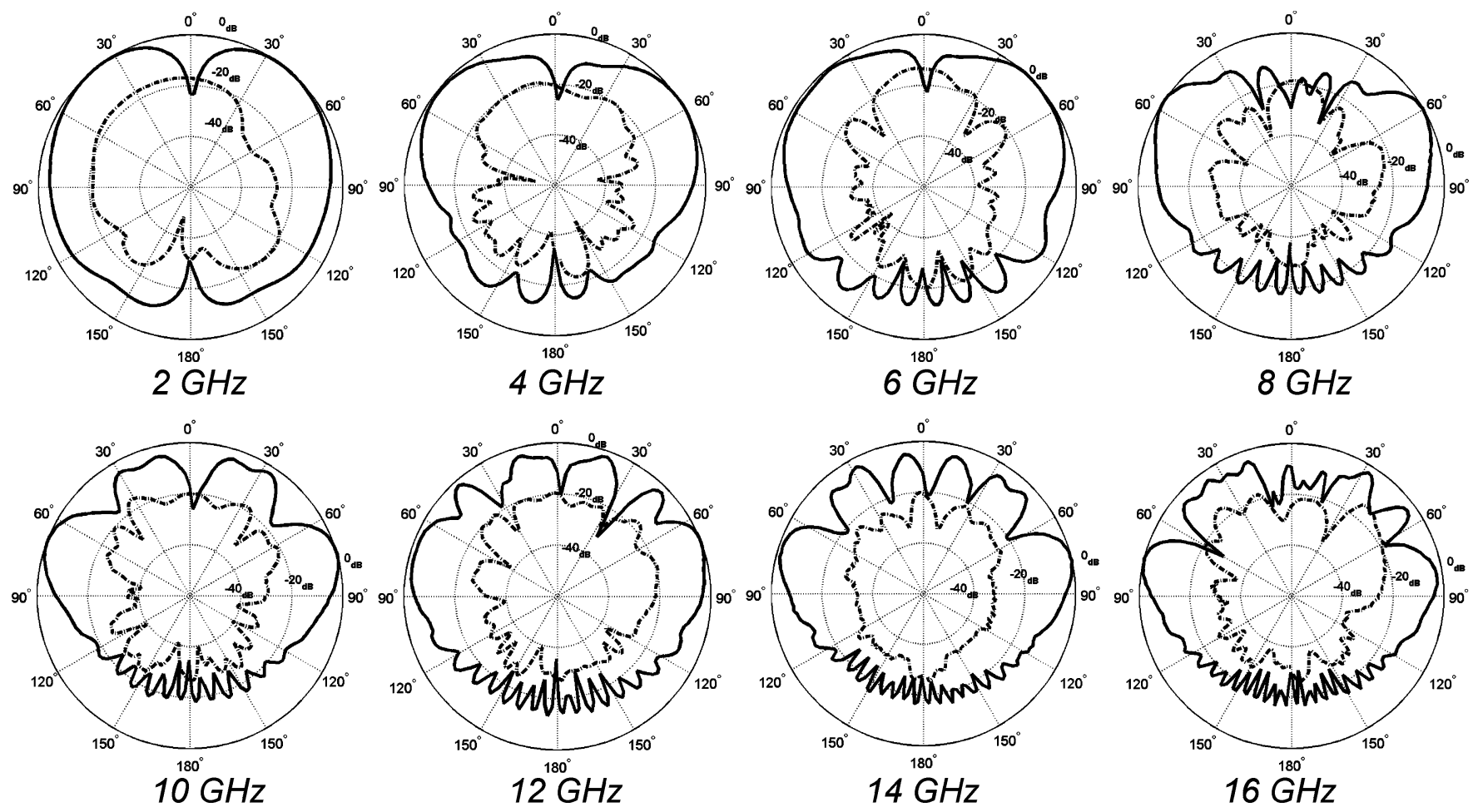

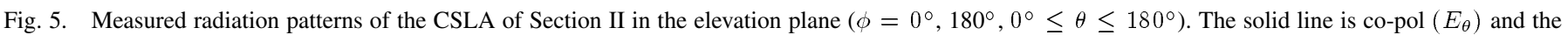
dash-dotted line is the cross-pol $\left(E_{\phi}\right)$ component.

$\alpha=60^{\circ}, R_{\mathrm{av}}=13.5 \mathrm{~mm}$, and three different arch thicknesses of $\tau=0.4,1.0$, and $1.6 \mathrm{~mm}$. The measured $S_{11}$ of these antennas are shown in Fig. 3(b). It is observed that thinner arch thicknesses provide a wider bandwidth. For the thinnest value of $\tau=0.4 \mathrm{~mm}$ an antenna with bandwidth of 3.7 to $11.6 \mathrm{GHz}$ is obtained.

\section{Radiation Parameters}

In the previous section, the optimum geometrical parameters of the antenna were experimentally obtained. Based on this process, a CSLA with $R_{\text {in }}=27.8 \mathrm{~mm}, R_{\text {out }}=28 \mathrm{~mm}$, and $\alpha=60^{\circ}$ is fabricated, on a $6 \mathrm{~cm} \times 3 \mathrm{~cm}$ substrate with $\epsilon_{r}=3.4$ and thickness of $0.5 \mathrm{~mm}$, and mounted on a $20 \mathrm{~cm} \times 20 \mathrm{~cm}$ ground plane. The dimensions are increased to lower the lowest and highest frequencies of operation and simplify the radiation pattern measurements. The antenna has a VSWR of lower than $2.2\left(S_{11}<-8.5 \mathrm{~dB}\right)$ from $1.78-14.5 \mathrm{GHz}$ (see Fig. 8), which is equivalent to an 8.5:1 impedance bandwidth. The radiation patterns of the antenna are measured across the entire frequency band and are presented in Figs. 4 and 5. Fig. 4 shows the far-field co- and cross polarized $\left(E_{\theta}, E_{\phi}\right)$ radiation patterns in the azimuth plane ( $x-y$ plane) for $\phi=0^{\circ}$ to $360^{\circ}$. It is shown that the radiation patterns remain similar up to about $f=8 \mathrm{GHz}$. As the frequency increases beyond $8 \mathrm{GHz}$, however, the radiation patterns start to change and show higher directivities in other directions.

Fig. 5 shows the measured radiation patterns of the antenna in the elevation plane at $\left(\phi=0^{\circ}, 180^{\circ}, 0^{\circ} \leq \theta \leq 180^{\circ}\right)$. The antenna patterns in another elevation plane $\left(\phi=90^{\circ}, 270^{\circ}\right.$, $0^{\circ} \leq \theta \leq 180^{\circ}$ ) are also measured and found to be similar to

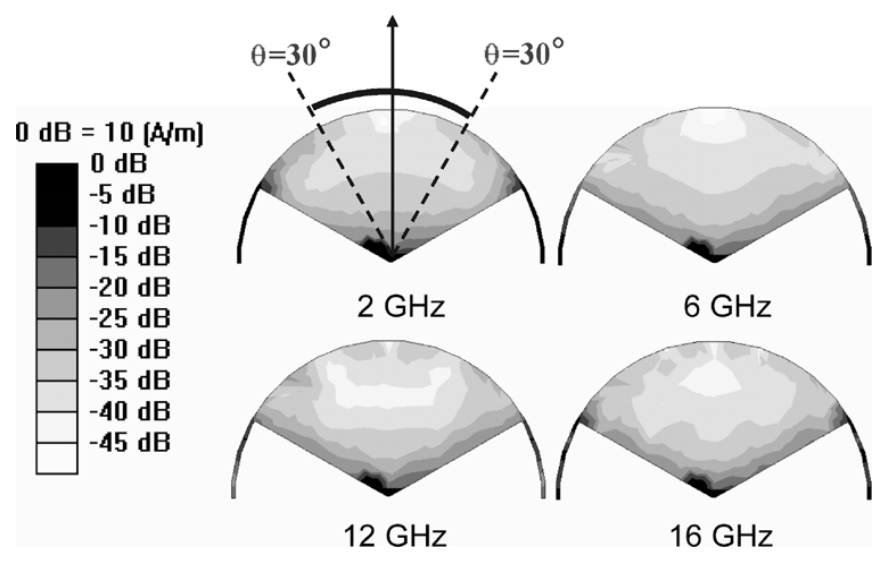

Fig. 6. Electric current distribution across the surface of the CSLA of Section II at four different frequencies.

those shown in Fig. 5. As frequency increases, the electrical dimensions of the antenna increase and as a result, the number of lobes increases. Also, the number of minor side lobes in the back of the ground plane $\left(90^{\circ} \leq \theta \leq 180^{\circ}\right)$ increases significantly. This is caused by diffractions from the edges of the ground plane, which has very large electrical dimensions at higher frequencies. At lower frequencies, the radiation patterns are symmetric; however, as frequency increases, the symmetry is not observed very well. This is caused by the presence of the coaxial cable that feeds the antenna and disturbs the symmetry of the measurement setup. Since the cable is electrically large at higher frequencies, a more pronounce asymmetry on the radiation patterns are observed at higher frequencies. In all the measured radiation patterns, the cross polarization level $\left(E_{\phi}\right)$ is 


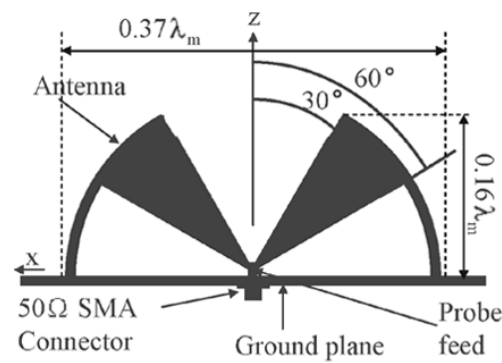

(a)

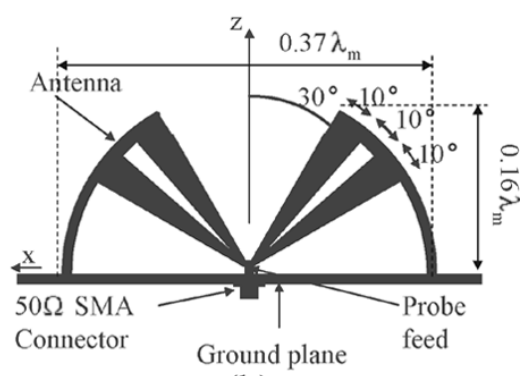

(b)

Fig. 7. Topology of the modified CSLAs of Section III. (a) M1-CSLA. (b) M2-CSLA.

shown to be negligible. This is an indication of good polarization purity across the entire frequency band.

\section{MODIFIED CSLAS}

In this section, we examine the possibilities of further reducing the size and weight of the CSLA by modifying its geometry. The CSLA demonstrated in the previous section was optimized to achieve the highest bandwidth allowing variation of only two independent parameters. Size reduction is very important for applications where the wavelength is large such as ground penetrating radars or VHF broadcast antennas. To examine ways to reduce the weight and size of the antenna, the current distribution over the metallic surfaces of the antenna is studied. The electric currents on the antenna surface is computed using a full-wave simulation tool based on the method of moments. The magnitude of the electric current on the surface of the antenna is shown in Fig. 6 at four different frequencies. It is noticed that the current magnitude is very small over a sector in the range of $0^{\circ} \leq \theta \leq 30^{\circ}$. This suggests that this sector of the antenna can be removed without significantly disturbing the CSLAs current distribution. This modification results in a design shown in Fig. 7(a) and, because of its similarity to the letter "M," this antenna will be referred to as the M1-CSLA. Applying the same approach to the M1-CSLA and examining the current distribution reveals that the electric current density is larger around the edges at $\theta=30^{\circ}, 60^{\circ}$ and has lower values in the area between $\left(30^{\circ}<\theta<60^{\circ}, \phi=0^{\circ}, 180^{\circ}\right)$. Therefore, two other pie-slice sections of the antenna, which are confined in the ranges $40^{\circ}<\theta<50^{\circ}$ for $\phi=0^{\circ}$ and $180^{\circ}$ can be removed to obtain the antenna shown in Fig. 7(b). For obvious reasons, this antenna is called the M2-CSLA. Prototypes of M1- and M2-CSLA with $\alpha=60^{\circ}, R_{\text {in }}=27.8 \mathrm{~mm}$ and $R_{\text {out }}=28 \mathrm{~mm}$ are fabricated on a thin substrate with thickness of $0.5 \mathrm{~mm}$ and dielectric constant of 3.4 and mounted on a $20 \mathrm{~cm} \times 20 \mathrm{~cm}$ square ground plane. The measured input reflection coefficients of these two antennas are given in Fig. 8 along with the $S_{11}$ of the original CSLA antenna with the same dimensions. It is seen that all of the antennas have VSWRs lower than 2.2 in a very large frequency band as shown in Table I. The best input match is, however, observed for the M2-CSLA with the VSWR lower than 2 across almost its entire band of operation.

The radiation patterns of the M2-CSLA are measured at three different planes at eight discrete frequency points and are presented in Figs. 9 and 10. The co-polarized, $E_{\theta}$, and cross-polarized, $E_{\phi}$, far-field radiation patterns at azimuth plane are shown

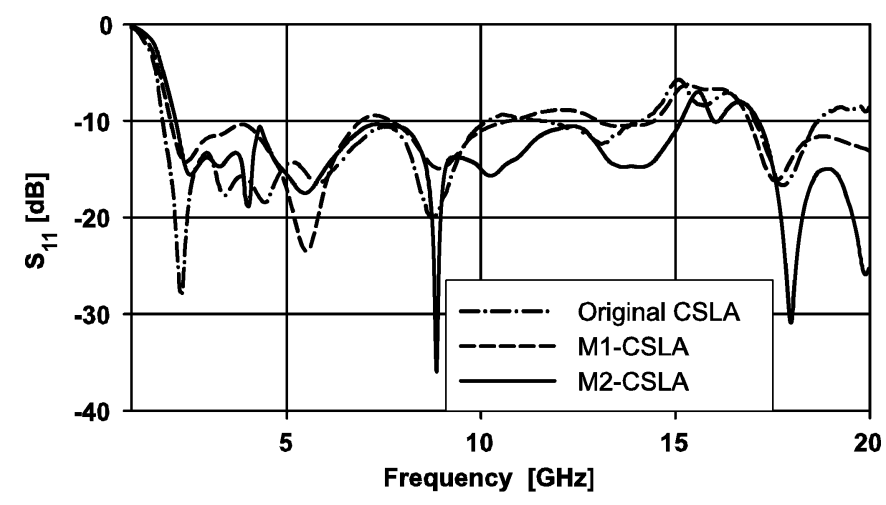

Fig. 8. Measured $S_{11}$ values of the original CSLA of Section II-C and the M1and M2-CSLAs of Section III.

TABLE I

COMPARISON BETWEEN THE BANDWIDTH OF THE ORIGINAL, M1-, AND M2-CSLAS

\begin{tabular}{c||c|c|c}
\hline Antenna Type & Frequency Range & BW & Highest VSWR \\
\hline Original CSLA & $1.7-14.5 \mathrm{GHz}$ & $8.50: 1$ & 2.2 \\
\hline M1-CSLA & $2-14.7 \mathrm{GHz}$ & $7.35: 1$ & 2.2 \\
\hline M2-CSLA & $2.05-15.3 \mathrm{GHz}$ & $7.46: 1$ & 2.2 \\
\hline
\end{tabular}

in Fig. 9 and are observed to be similar to those of the original CSLA. The elevation patterns measured in the $x-z$ plane $\left(\phi=0^{\circ}, 180^{\circ}, 0^{\circ} \leq \theta \leq 180^{\circ}\right)$ are presented in Fig. 10. The elevation patterns in the $y-z$ plane $\left(\phi=90^{\circ}, 270^{\circ}\right.$, $0^{\circ} \leq \theta \leq 180^{\circ}$ ) are also measured and found to be similar to those shown in Fig. 10. It is observed that, similar to the original CSLA, the minor side lobe levels increase with frequency.

The gains of the three CSLAs are measured in the anechoic chamber of the University of Michigan in the frequency range of $f=2-16 \mathrm{GHz}$ using a double-ridge standard horn reference antenna and are presented in Fig. 11. The gain of the antennas are measured at $\phi=90^{\circ}, \theta=90^{\circ}$. As frequency increases, the electrical dimensions of the antenna increases and therefore the antenna gain should increase. However, as is observed from Fig. 11, the antenna gain decreases as frequency increases from 6 up to $10 \mathrm{GHz}$. This is a consequence of the change in the direction of maximum radiation in the azimuth plane as is observed from Figs. 4 and 9. The performance of UWB systems is affected by the variations in the gain and radiation patterns of its antennas. Even if the antenna gains do not change as a function of frequency, frequency dependent variations in path loss and 

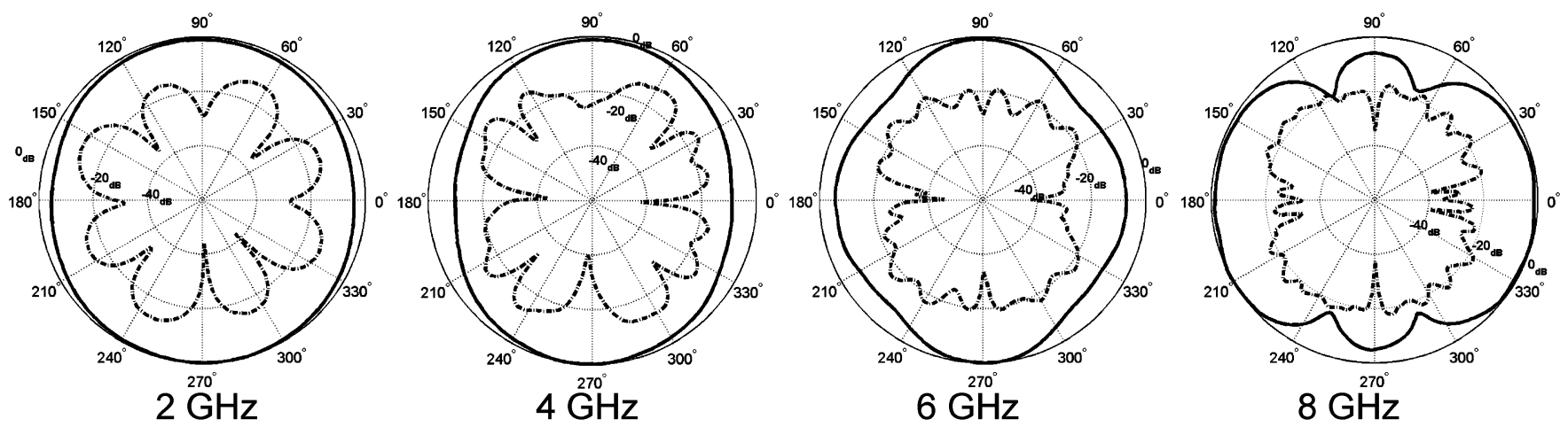

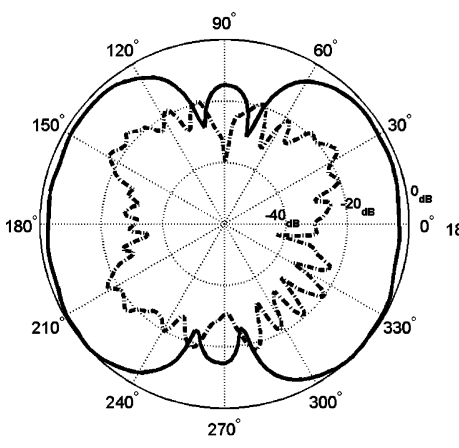

$10 \mathrm{GHz}$

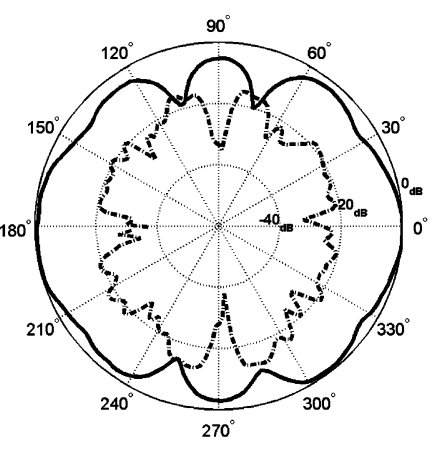

$12 \mathrm{GHz}$

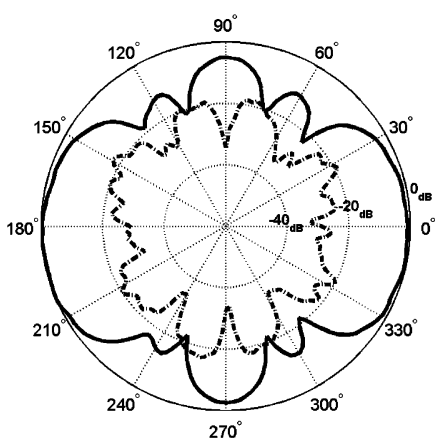

$14 \mathrm{GHz}$

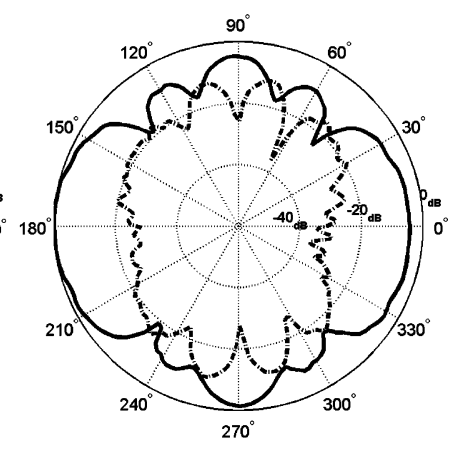

$16 \mathrm{GHz}$

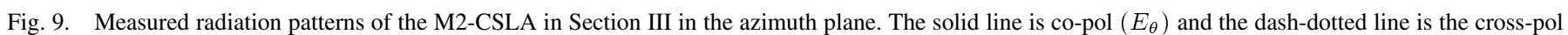
$\left(E_{\phi}\right)$ component.
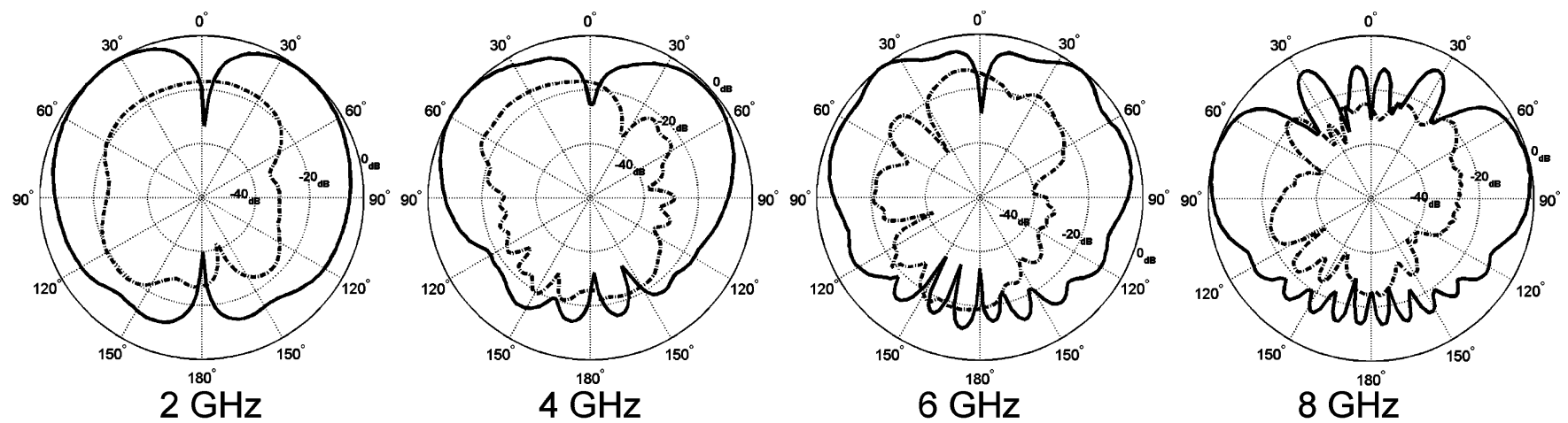

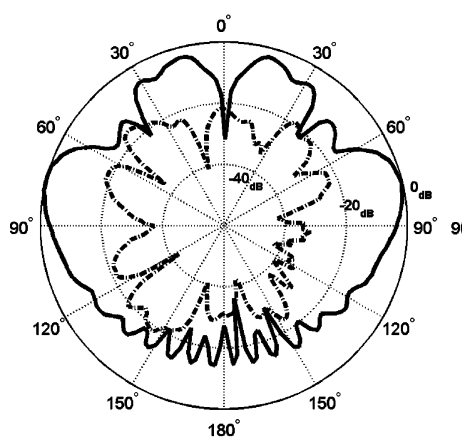

$10 \mathrm{GHz}$

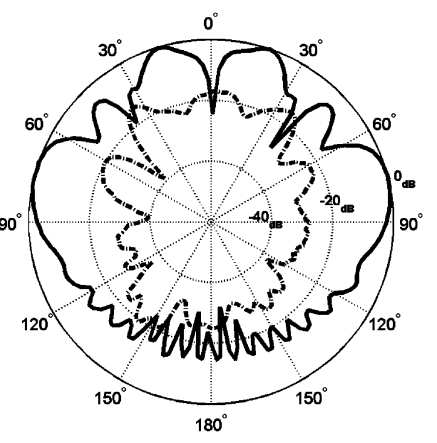

$12 \mathrm{GHz}$

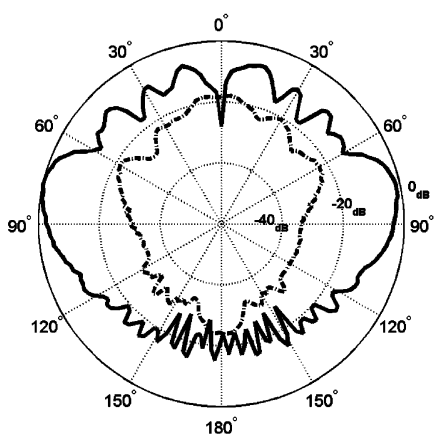

$14 \mathrm{GHz}$

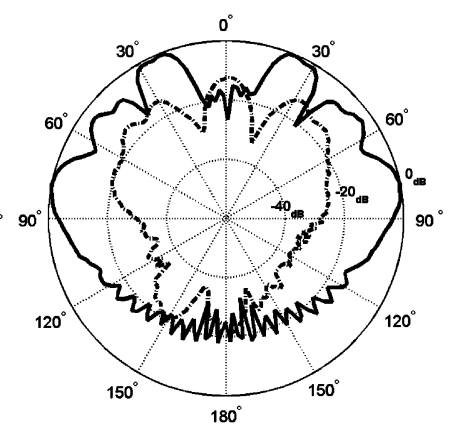

$16 \mathrm{GHz}$

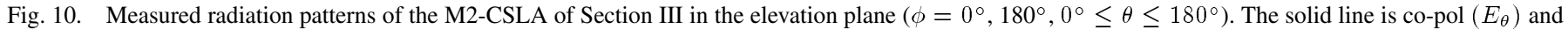
the dash-dotted line is the cross-pol $\left(E_{\phi}\right)$ component.

other system components exist and their effects must be taken into account. However, once these variations are modeled (e.g., antenna gains as a function of frequency are measured), their effect can be taken into account as described in detail in [14].

\section{Time Domain Measurements}

As shown in the previous section the proposed antennas, and particularly the original CSLA, present a very wide bandwidth. 


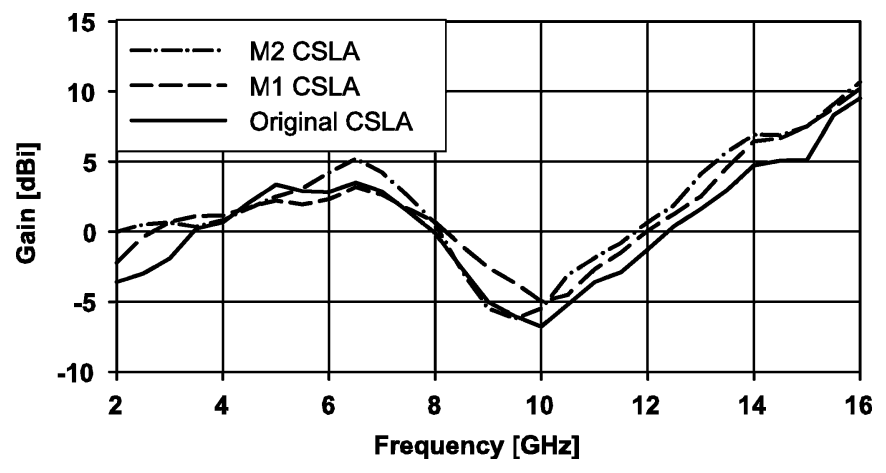

Fig. 11. Measured gain of the original CSLA of Section II-C and the M1and M2-CSLAs of Section III. The gains are measured in the azimuth plane at $\left(\phi=90^{\circ}, \theta=90^{\circ}\right)$.

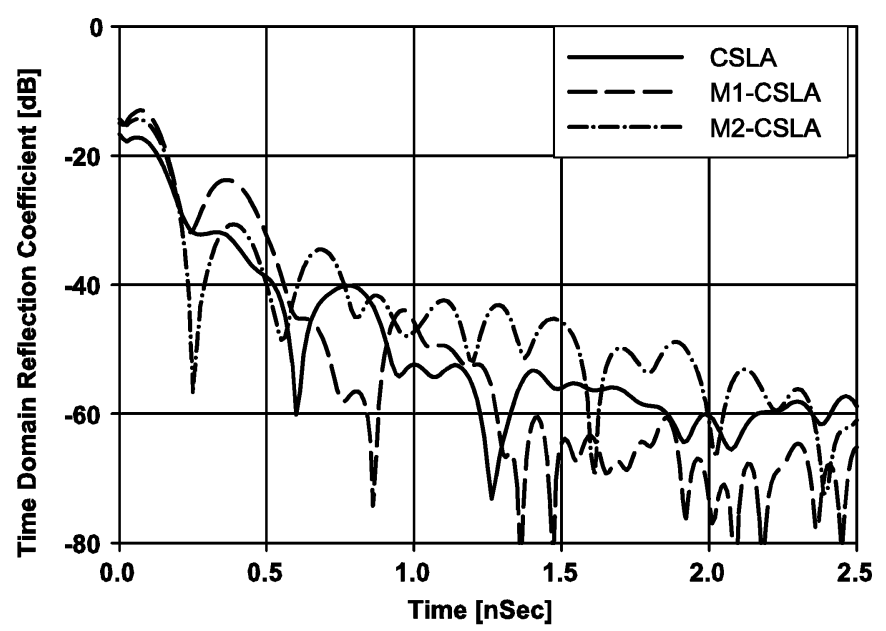

(a)

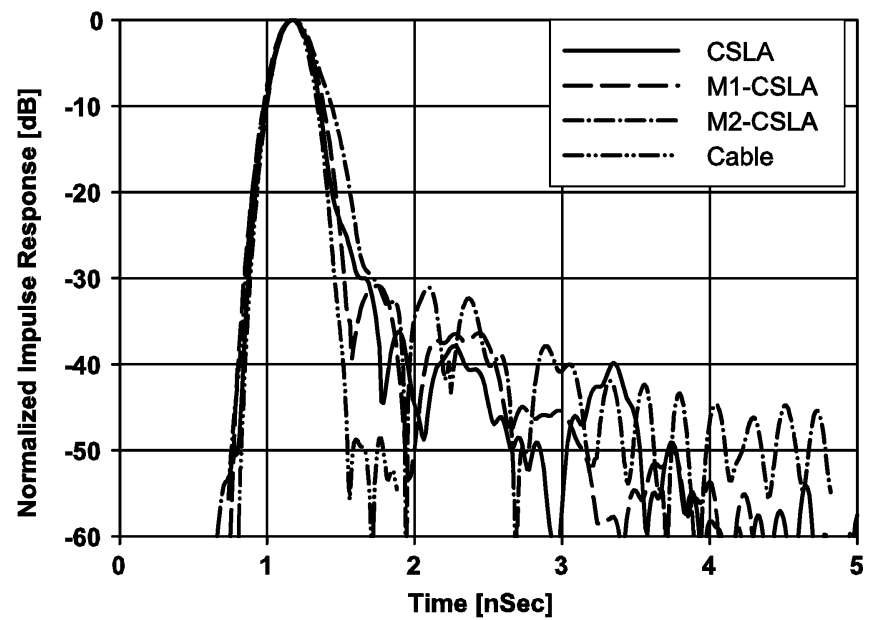

(b)

Fig. 12. (a) Time domain reflection coefficients, $|\Gamma|$, of the original CSLA of Section II-C and the M1- and M2-CSLAs of Section III. (b) Time domain impulse response of the system consisting two identical CSLAs.

However, having a wide-band frequency-domain response does not necessarily ensure that the antenna behaves well in the time domain as well; that is, a narrow time-domain pulse is not widened by the antenna. Some multiresonant wide-band antennas, such as log-periodic antennas, due to multiple reflections within the antenna structure, widen a narrow pulse in time domain. Therefore, in order to ensure the usefulness of the proposed antenna for time-domain applications, the time-domain responses of the antennas must also be examined. The time-domain reflection coefficients of the CSLAs, are measured using the time-domain capabilities of an HP8720B network analyzer. The network analyzer calculates the time-domain response by measuring the wide-band frequency-domain response (in this case in the $2-12 \mathrm{GHz}$ band) and calculating its inverse Fourier transform to obtain an approximation of the impulse response of the network. Fig. 12(a) shows the time-domain variations of the reflection coefficients, $|\Gamma|$, of these three antennas in logarithmic scale. It is observed that all of the antennas have a reflection at $t=0 \mathrm{~ns}$, which corresponds to the discontinuity at the plane of calibration (input of the SMA connector). The peak reflection at $t=80 \mathrm{ps}$ corresponds to the probe-antenna transition. The M2-CSLA shows stronger small reflections, which is a consequence of the larger number of discontinuities in its structure.

In addition to the input reflection coefficient, the time-domain impulse responses of a system that contains two identical CSLAs (Original CSLA, M1-CSLA, or M2-CSLA) are also measured. The measurement setup consists of two identical CSLAs that are placed $30 \mathrm{~cm}$ apart and parallel to each other inside a small anechoic chamber. The antennas are connected to the two ports of a calibrated vector network analyzer that is located outside the chamber and the time-domain transmission coefficients $\left(S_{21}\right)$ of the system is measured using the VNA. The normalized time-domain impulse response of this system is shown in Fig. 12(b) along with the time-domain impulse response of a coaxial cable with the same electrical length as a reference. It can be seen that the multiple small peaks that come after the main peak are at least $30 \mathrm{~dB}$ lower in magnitude than the main peak. It is also observed that the original CSLA and the M1-CSLA have a response that is closer to that of the coaxial cable (which ideally should be a delta function) than the impulse response of the M2-CSLA. The time-domain impulse response of the M2-CSLA is more distorted and has stronger multiple reflections, which are caused by the larger number of discontinuities in its structure.

\section{CONCLUSION}

A novel UWB CSLA is designed and is shown to have a 8.5:1 impedance bandwidth. The antenna has consistent radiation parameters over a 4.5:1 frequency range with excellent polarization purity over the entire 8.5:1 frequency range. Modified versions of this antenna, with reduced metallic surfaces and similar radiation parameters, were also designed, fabricated, and measured. Furthermore, the time domain impulse-response measurement results show that the antenna does not significantly distort the temporal response of a signal whose spectral content does not fall outside the operating band of antenna.

\section{REFERENCES}

[1] G. F. Ross, "The Transient analysis of multiple beam feed networks for array systems," Ph.D. dissertation, Polytechnic Institute of Brooklyn, Brooklyn, NY, 1963.

[2] C. A. Balanis, Antenna Theory: Analysis and Design, 2nd ed. New York: Wiley, 1996, ch. 12 
[3] C. H. Walter, Traveling Wave Antennas: Peninsula Pub., 1990.

[4] R. G. Corzine and J. A. Mosko, Four Armed Spiral Antennas. Norwood, MA: Artech House, 1990.

[5] Y. Mushiake, Self-Complementary Antennas: Principle of Self-Complementarity for Constant Impedance: Springer, 1996.

[6] G. Kumar and K. Ray, Broad-band Microstrip Antennas. Norwood, MA: Artech House, 2003.

[7] K. L. Wong, Compact and Broad-band Microstrip Antennas. New York: Wiley, 2002.

[8] N. Behdad and K. Sarabandi, "A wide-band slot antenna design employing a fictitious short circuit concept," IEEE Trans. Antennas Propag., vol. 53, no. 1, pp. 475-482, Jan. 2005.

[9] - "A multiresonant single-element wide-band slot antenna," IEEE Antennas Wireless Propag. Lett., vol. 3, pp. 5-8, 2004.

[10] N. P. Agrawal, G. Kumar, and K. P. Ray, "New wide-band monopole antennas," in Proc. IEEE Antennas and Propagation Soc. Int. Symp., vol. 1, Jul. 13-18, 1997, pp. 248-251.

[11] M. J. Ammann and Z. J. Chen, "Wideband monopole antennas for multiband wireless systems," IEEE Antennas Propag. Mag., vol. 45, pp. 146-150, 2003.

[12] M. Lapierre, Y. M. M. Antar, A. Ittipiboon, and A. Petosa, "A wide-band monopole antenna using dielectric resonator loading," in Proc. IEEE Antennas and Propagation Soc. Int. Symp., vol. 3, Jun. 22-27, 2003, pp. 16-19.

[13] IE3D Electromagnetic Simulation and Optimization Software, Zeland Software, Inc..

[14] J. D. Taylor, Introduction to Ultra Wide-band Radar Systems, 1st ed. Boca Raton, FL: CRC Press, 1995, ch. 5, pp. 147-162.

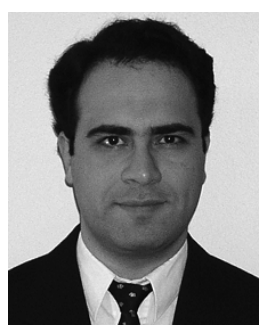

Nader Behdad (S'98) was born in Mashhad, Iran, in 1977. He received the Bachelor of Science and Master of Science degrees from Sharif University of Technology, Tehran, Iran, and the University of Michigan, Ann Arbor, MI, in 2000 and 2003 respectively. He is currently working toward the Ph.D. degree in the Department of Electrical Engineering and Computer Science at the University of Michigan where he is working on bandwidth enhancement and miniaturization of printed antennas.

From 2000 to 2001, he was with the Electronics Research Center at Sharif University of Technology as an Antenna Design Engineer working on the design of antennas for wireless local loop (WLL) systems. Since January 2002, he has been working as a Research Assistant in the Radiation Laboratory of the University of Michigan.

Mr. Behdad received the Best Student Paper Award at the Antenna Applications Symposium held in Monticello, IL, in September 2003, he won second place at the student paper competition of the USNC/URSI Conference in Boulder, CO, in January 2004, and he was the recipient of the Horace $\mathrm{H}$. Rackham Predoctoral Fellowship from the University of Michigan in 2005.

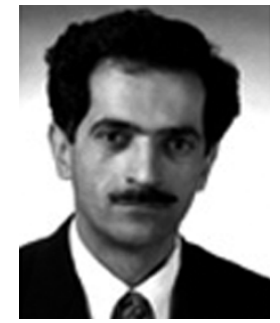

Kamal Sarabandi (S'87-M'90-SM'92-F'00) received the B.S. degree in electrical engineering from Sharif University of Technology, Tehran, Iran, in 1980, the M.S. degree in electrical engineering/mathematics, and the Ph.D. degree in electrical engineering from The University of Michigan, Ann Arbor, in 1986 and 1989, respectively.

$\mathrm{He}$ is Director of the Radiation Laboratory and a Professor in the Department of Electrical Engineering and Computer Science, The University of Michigan. He has 20 years of experience with wave propagation in random media, communication channel modeling, microwave sensors, and radar systems and is leading a large research group consisting of four research scientists, 12 Ph.D. students, and two M.S. students. Over the past ten years he has graduated $20 \mathrm{Ph} . \mathrm{D}$. students. He has served as the Principal Investigator on many projects sponsored by NASA, JPL, ARO, ONR, ARL, NSF, DARPA, and numerous industries. He has published many book chapters and more than 115 papers in refereed journals on electromagnetic scattering, random media modeling, wave propagation, antennas, microwave measurement techniques, radar calibration, inverse scattering problems, and microwave sensors. He has also had more than 230 papers and invited presentations in many national and international conferences and symposia on similar subjects. His research areas of interest include microwave and millimeter-wave radar remote sensing, electromagnetic wave propagation, and antenna miniaturization.

Dr. Sarabandi is a Member of the International Scientific Radio Union (URSI) Commission F and of The Electromagnetic Academy. He received the Henry Russel Award from the Regent of The University of Michigan (the highest honor the University of Michigan bestows on a faculty member at the assistant or associate level). He received a 1996 Teaching Excellence Award from the Department of Electrical Engineering and Computer Science and a 1999 GAAC Distinguished Lecturer Award from the German Federal Ministry for Education, Science, and Technology, given to about ten individuals worldwide in all areas of engineering, science, medicine, and law. He also received the 2003/2004 College of Engineering Research Excellence Award, The University of Michigan. In the past several years, joint papers presented by his students at a number of symposia (IEEE AP'95,'97,'00,'01,'03 IEEE IGARSS'99,'02, IEEE MTTS'01) have received student prize paper awards. $\mathrm{He}$ is a Vice President of the IEEE Geoscience and Remote Sensing Society (GRSS), a past Chairman of the Awards Committee of the IEEE GRSS from 1998 to 2002, and a Member of the IEEE Technical Activities Board Awards Committee from 2000 to 2002. He is an Associate Editor of the IEEE Transactions on ANTENNAS and PRopagation and the IEEE SENSORS JOURNAL. He is listed in American Men \& Women of Science, Who's Who in America, and Who's Who in Electromagnetics. 